DOI: $10.21767 / 2254-6758.100106$

\section{Hand Revascularization in the Paediatric Patient}

\section{Abstract}

The current case report will focus on the key factors that influence the revascularization of a hand. Emphasis will be placed on microsurgery, timing factors and intraoperative factors as well as postoperative factors.

The principles of microsurgery in the paediatric patient compared to that of the adult are still the same. Paediatric patients do pose an advantage of having no/ fewer comorbidities. It has been shown they have a high capacity of regeneration; however paediatric patients do come with their challenges such as the size and diameter of the vessels.

This case presentation will focus on a 6-year-old male child's dominant hand revascularization, from onset of injury to rehabilitation phase. Timing is crucial, as if not picked up early enough it is associated with morbidity and mortality. We also look at the successful outcome of the revascularization surgery in the paediatric patient.

Keywords: Emergency; Hand surgery; Microsurgery; Reconstruction; Paediatrics; Re-vascularization; Vascular surgery

Received: June 25, 2018; Accepted: June 29, 2018; Published: July 04, 2018

\section{Palesa Phalafala*, Dimitri Liakos, Viwe Nogaga and Chrysis Sofianos}

\begin{abstract}
Department of Plastic and Reconstructive surgery, University of the Witwatersrand, Charlotte Maxeke Johannesburg Academic Hospital, Johannesburg, South Africa
\end{abstract}

Corresponding author: Palesa Phalafala

$\equiv$ palesa@phalafala.com

Department of Plastic and Reconstructive surgery, University of the Witwatersrand, Charlotte Maxeke Johannesburg Academic Hospital, Johannesburg, South Africa.

Tel: +27845807454

Citation: Phalafala P, Liakos D, Nogaga V, Sofianos C (2018) Hand Revascularization in the Paediatric Patient. J Univer Surg. Vol.6 No.2:16

\section{Background}

In the light of this case presentation that is to follow, it is important for health care providers to understand critical steps in the management of a devascularized limb. Each phase of treatment is vital for optimum functionality of the limb in the long run.

Aboelatta et al. described free tissue transfer in twenty-eight paediatric patients under the age of 15 years old, where twentyone $(75 \%)$ were elective cases and seven (25\%) patients were emergency cases [1]. Their results showed that over the age of 10 years, the success/failure and complication rates are similar to that of adults. Between 5 and 10 years old posed a higher success rate compared to the age group below 5 years old.

\section{Case Presentation}

DP, a 6yr old boy with no comorbidities, was transferred from a Secondary Hospital in Johannesburg with a history of being injured by a construction machine, which he accidentally switched on whilst playing. His left hand was lacerated at the level of the wrist joint (proximal wrist crease). This occurred at approximately $10 \mathrm{~h} 00 \mathrm{am}$. At the scene, DP's father wrapped the hand in a shirt to prevent further bleeding and took him to the nearest hospital.

At the Secondary hospital, the patient was wrongly triaged as a non-urgent case. Once seen by the doctors, he was diagnosed as a tendon injury with questionable vascular compromiso (Figure 1.1). He was then referred to a quaternary hospital for further management. By the time he arrived, it was 8 hours post injury. Clinically the patient was haemodynamically stable. The appearance of the hand was dusky and blue; both ulnar and radial pulse were impalpable and the child was evidently in pain. Resuscitation with an intravenous line was placed (in the unaffected arm) and analgesia commenced. A baseline X-ray demonstrated no fractures (Figure 1.2).

\section{Treatment}

\section{Pre-operatively}

- The patient was cleared as per ATLS principles.

- The parents were counseled and consented for revascularization. 


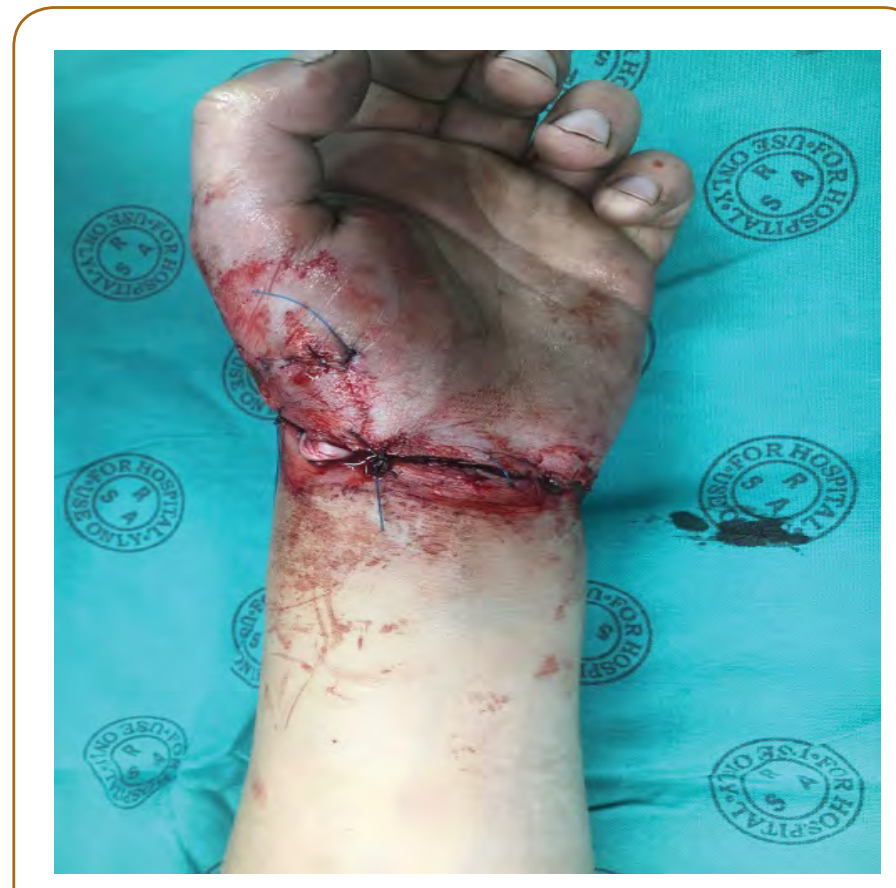

Figure 1.1 Diagnosed as a tendon injury.

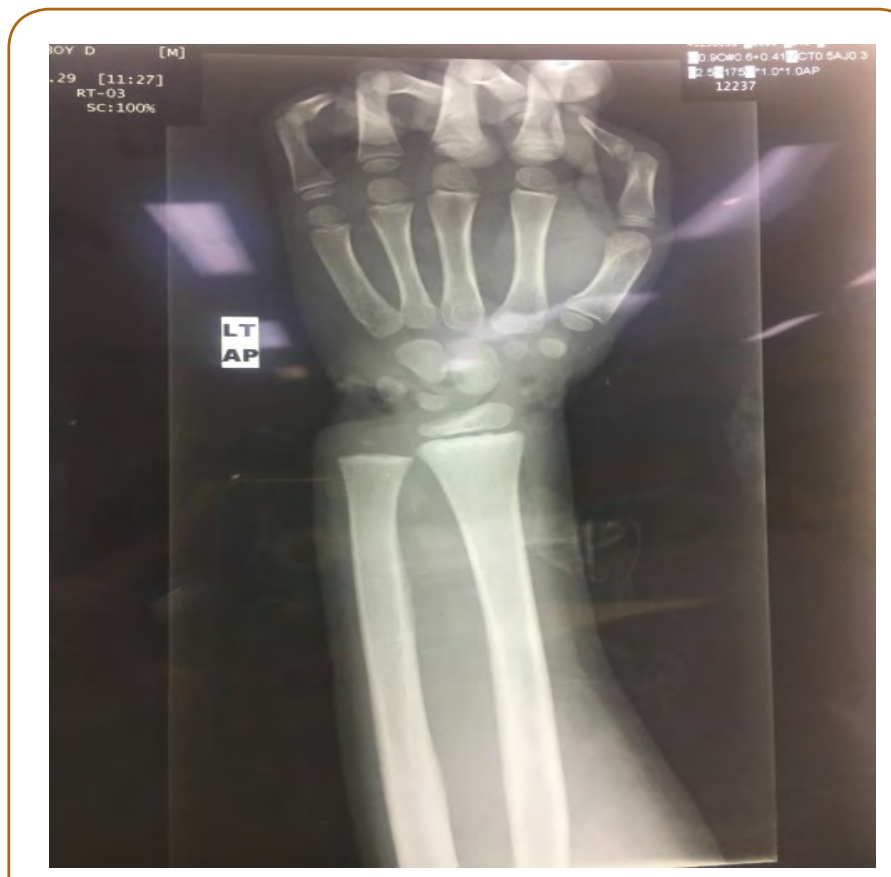

Figure 1.2 A baseline X-ray demonstrated no fractures.

- Blood products were ordered on standby (type and screen).

- The patient was booked for emergency surgery.

\section{Intra-operatively}

- General anaesthesia was used.

- Thorough examination of the left hand was done under anaesthesia (Figure 2.1), which concluded that there was disarticulation of the wrist joint, the radial artery was degloved and ulnar artery was lacerated at the level of the wrist. The following structures were identified as being intact: Flexor carpi ulnaris, Flexor carpi radialis, Flexor digitorum profundus together with Flexor digitorum superficialis to the index, middle, ring, and little finger. The ulnar and radial nerve were also found to be intact.

- The use of a tourniquet is prohibited in revascularization surgery.

- The great saphenous vein, from the right leg, was harvested as a graft to approximate the ulnar artery.

- Anastomosis was done under microscope (Carl Zeiss f $170)$, with nylon 10.0 interrupted sutures $[2,3]$.

- Heparinized saline was used for irrigation of the anastomosis with a concentration of 20000 units of unfractionated heparin in $200 \mathrm{ml}$ normal saline, $100 \mathrm{U} /$ $\mathrm{ml}$ (Figure 2.2) [4]. Intraoperatively, 2\% lignocaine was irrigated onto the vessels to diminish vasospastic events.

- The veins on the volar aspect of the hand were severed and not repaired, leaving the dorsal veins draining the hand. The dorsal veins were not tampered with.

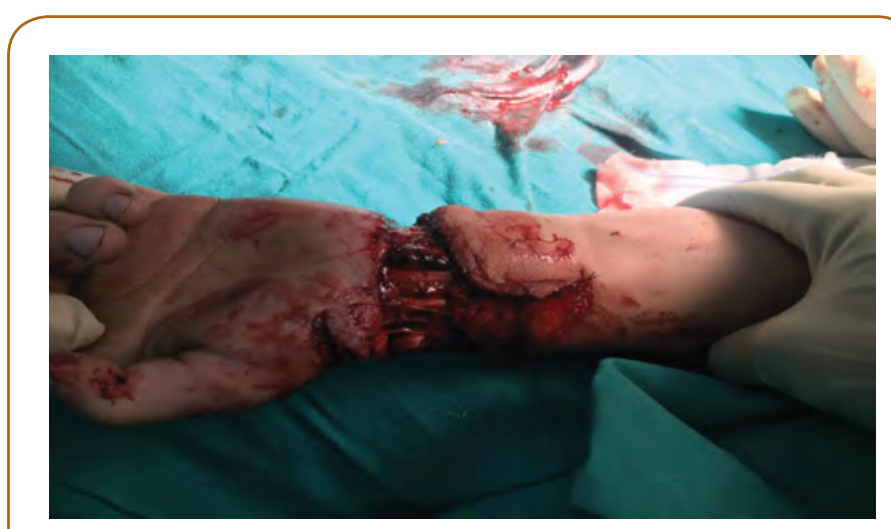

Figure 2.1 Thorough examination of the left hand was done under anaesthesia.

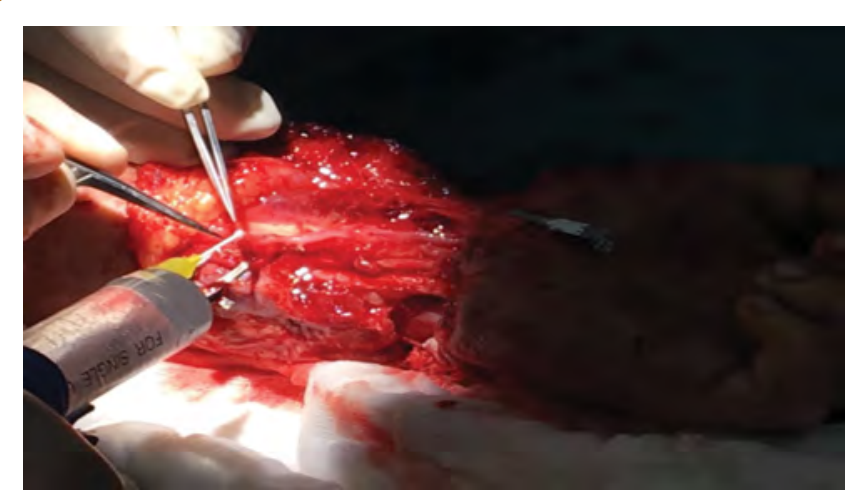

Figure 2.2 Unfractionated heparin in $200 \mathrm{ml}$ normal saline, 100 $\mathrm{U} / \mathrm{ml}$. 


\section{Post- operatively}

- The patient was placed in a Paediatric Intensive Care Unit for 24 hours.

- A bulky dressing was used for 72 hours post operatively.

- A re-look exploration was done within 48 hours post initial operation and it was noted that the hand was perfusing well through the competent graft. The median nerve was found to be intact at this point (Figure 3).

During his admission on the ward, the patient acquired a Pseudomonas aeruginosa infection at the wound site. Acetic acid wash was performed daily with a honey based bacteriostatic dressing (Melladerm, Medika SA) until the infection was cleared [5-8].

At 25 days post-initial operation, sensation was intact. In terms of motor function, all fingers had good range of motion. A split thickness skin graft was placed on the healthy granulated wounds and the external fixator was removed (Figure 4).

Meticulous rehabilitation was done where the patient was seen by the occupational therapy team, for muscle strength, joint mobility, and improved hand function. A month post injury, the child was seen in the outpatient department and the wounds were closed and healing well and hand function was thoroughly assessed (Figure 5).

In terms of functionality of the hand, which was assessed by occupational paediatric therapist, he is able to grip objects however according to the oxford muscle strength grading scale done all on active movement (Table 1): his wrist flexion was $3 / 5$ and extension was $2 / 5$. The metacarpal phalangeal joint was $2 / 5$ on both flexion and extension. The distal and proximal phalangeal

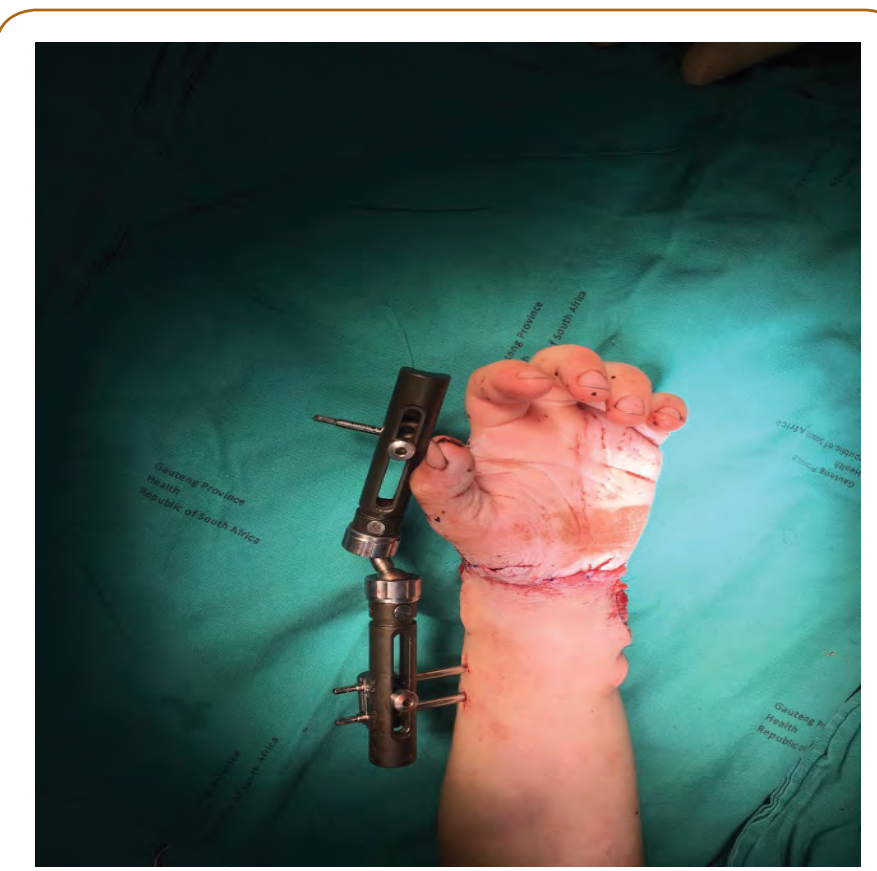

Figure 3 The median nerve was found to be intact at this point.

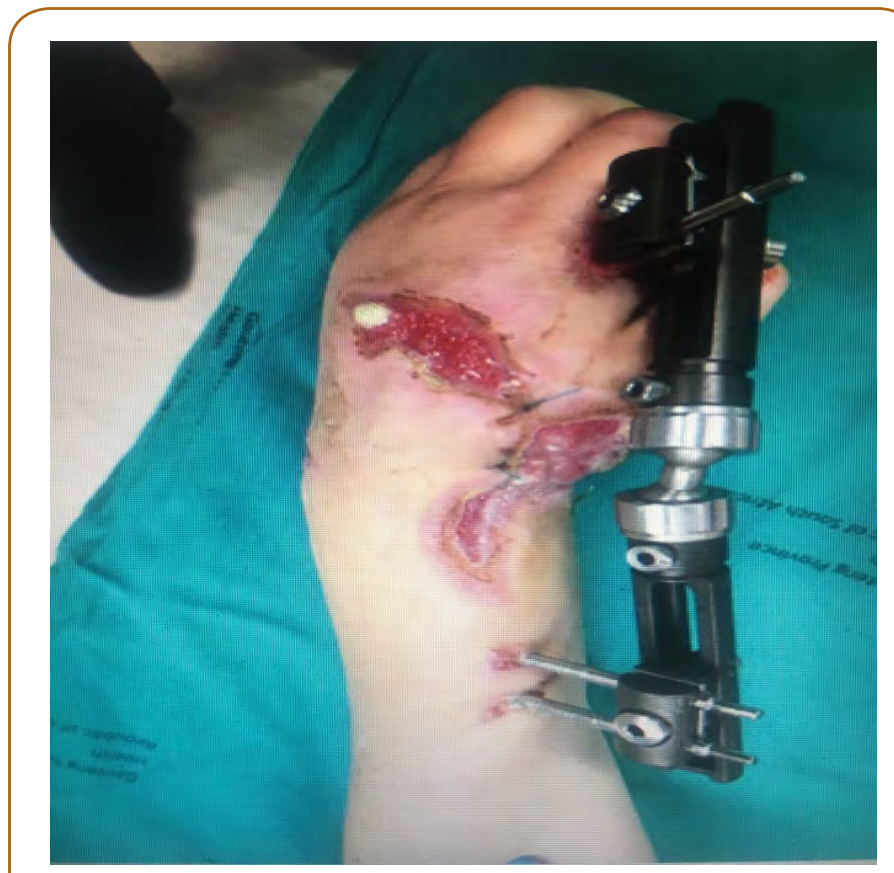

Figure 4 The external fixator was removed.

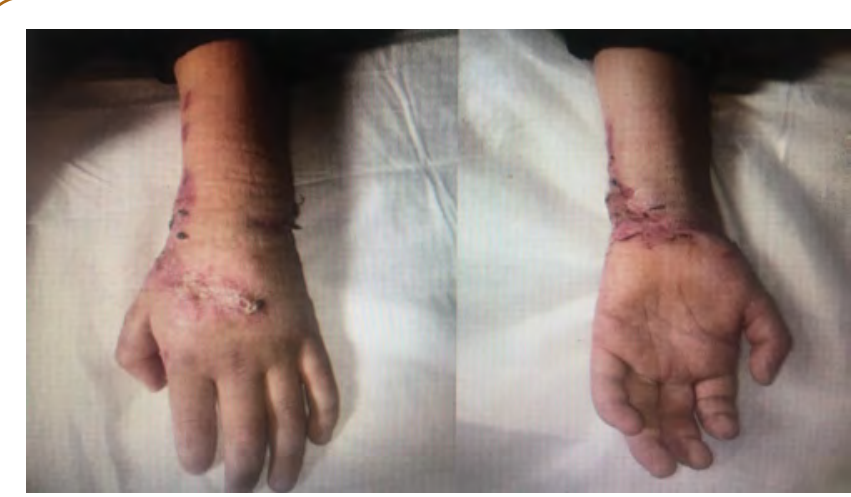

Figure 5 The child was seen in the outpatient department and the wounds were closed and healing well.

Table 1: Oxford muscle strength grading scale.

\begin{tabular}{cc} 
& Oxford Strength Grading Scale \\
\hline $0 / 5$ & No Contraction \\
\hline $1 / 5$ & Visible/Palpable muscle contraction with no movement \\
\hline $2 / 5$ & Movement with gravity eliminated \\
$3 / 5$ & Movement against gravity only \\
\hline $4 / 5$ & Movement against gravity with some resistance \\
\hline $5 / 5$ & Movement against gravity with full resistance \\
\hline
\end{tabular}

joints were $4 / 5$. In terms of range of motion which was measured with a goniometer the wrist had $20^{\circ}$ active flexion and $15^{\circ}$ of passive flexion. He was unable to oppose the thumb and little finger but he palmar prehension was present (Figure 6). He is able to do activities of daily living such as: bathing, getting dressed, eating and transferring objects. It was noted that bilateral hand tasks such as tying shoelaces were done with a bit of difficulty. In conclusion active range of motion of the wrist and metacarpal 


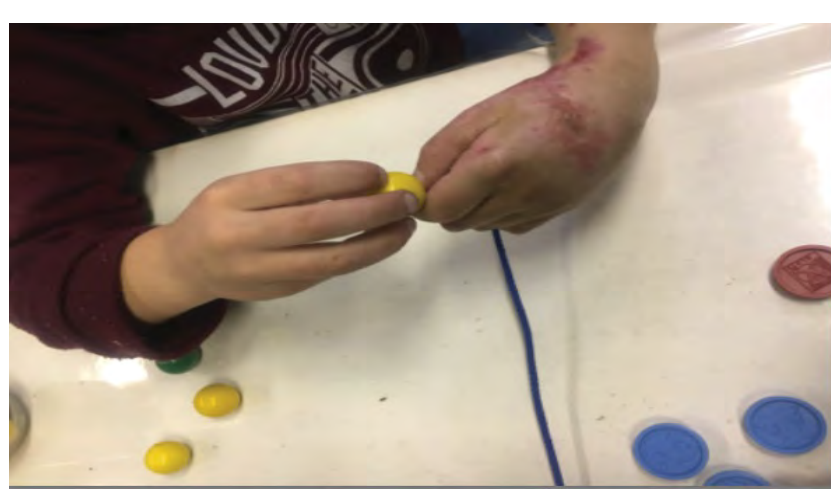

Figure 6 He was unable to oppose the thumb and little finger but the palmar prehension was present.

phalangeal joints were decreased comparatively looking at the uninjured hand however pain from scar tissue over these joints could have contributed to the range of motion as passive motion was the same as the uninjured hand. He is currently undergoing aggressive hand therapy and scar management with the occupational therapy team.

\section{Discussion}

The objective in revascularization is a viable hand [9]. In paediatric patients, it markedly assists in neurological skill development, to help with psychosocial and activities of daily living [10]. Revascularization surgery tries to regain hand function to a near normal state [9].

Minimizing ischaemic time is crucial when revascularization surgery is to be performed. Skin and subcutaneous issue tolerate a warm ischaemic time of 6 hours and cold ischaemic time of 12 hours, whereas muscle tolerates warm ischaemic time of 3 hours and cold ischaemic time of 8 hours [8].

Salvage techniques include: 1) Placing the distal part of the limb in close proximity of a plastic bag filled with ice. The aim is to keep the distal part cold to prevent the dangerous consequences of warm ischaemia, however not in direct contact with the ice to prevent frostbite $[4,8]$. 2) Grafting the arteries where possible should be done rather than anastomosing a vessel under tension 3) A second sitting, 48 hours post initial repair should be planned for repair of tendons and nerves where indicated. Relook operations/procedures are not mandatory but depending on the state of the patient and/or the state of the injury, you might be compelled to repair the arteries and come back for a secondary procedure [11-13]. Clear documentation is crucial post initial operation as some structures may not be lying in their usual anatomical positions and may be accidentally injured during relook operations [13].

The team should be lead by an experienced micro surgeon with assistance from a multidisciplinary team. This is pivotal for a successful outcome [4].

O'Brien et al. reported in 1980 a case series of 18 digital replantation's and 13 revascularizations in 27 children with a mean age of 6.5 years. The overall digit survival was $65 \%$ and three factors-the age of the child, the level of injury and the type of injury determined the likelihood of success [14].

In view of this case study, factors such as age and mechanism of injury (clean laceration versus a crush injury) showed a better prognostic outcome for the patient $[11,14,15]$.

On arrival to the emergency department life-threatening injuries always take precedence and adequate resuscitation is important to stabilize the patient for surgery [12]. Actively bleeding vessels can be controlled with pressure dressings. Blind attempts at suturing and clamping vessels is not recommended, as harm can be sustained to healthy vessels and nerves.

The following medication should be administered: 1) Tetanus toxoid if the immunization status of the child is uncertain or if the last dose was given more than 5 years previously 2) Prophylactic antibiotics are strongly indicated in amputated limbs $[4,15]$.

Intraoperatively, neurovascular structures are identified (proximal and distal ends) and assessed as to whether they can be repaired. No structure should be repaired under tension; grafts should be harvested in this instance. All structures that are devitalized should be sharply debrided at their ends [4]. Removal of intravascular clots and debris with heparinized saline is crucial prior to repair. Vein grafts, for arterial repair, can be harvested from the contralateral arm or lower limbs. The graft should be placed in reverse as to not hinder the flow of blood from the venous valves [5]. Once the artery has been primarily repaired or anastomosed with a graft, time should be taken to assess for perfusion of the distal part. This can be achieved by assessing venous flow from the distal limb, examining the colour change, and pricking the distal part with a $22 \mathrm{G}$ needle to see if bright red blood seeps through $[4,15]$.

Post-operatively there is a critical period in which a thrombus can form in the anastomosis, which is the first 72 hours of healing. If a thrombus forms and is not cleared, the anastomosis will fail $[4,6]$. It is essential that external factors such as adequate blood pressure, temperature and anxiety be addressed. Hypotension, hypothermia and an increased adrenergic state cause vasoconstriction and precipitate in failure in the graft $[4,8,15]$.

\section{Conclusion}

Success in revascularization surgery is measured by the patient's functionality post operatively; to ensure the limb is not a burden [15]. The patient and parents were very pleased of the outcome of the surgery, as their main concern was functionality postoperatively. The child was able to return to his regular schooling program one and a half months post-operation, although he still complained of slight stiffness around the metacarpal joints. He still continues with a vigorous occupational therapy schedule, and is able to perform the majority of his activities of daily living.

\section{Consent}

Written consent was obtained from the patient's parents for publishing (Supplementary attached). 


\section{References}

1 Aboelatta YA, Aly HM (2013) Free tissue transfer and replantation in pediatric patients. J Hand Microsurg 5: 74-80.

2 Serletti JM (2005) Current trends in pediatric microsurgery. Clin Plast Surg 32: 45-52.

3 Tark KC, Kim YW, Lee YH, Lew JD (1989) Replantation and revascularization of hands: Clinical analysis and functional results of 261 cases. J Hand Surg Am 14: 17-27.

4 Charles HT (2014) Grabb \& Smith: Plastic surgery, $7^{\text {th }}$ edn, NY: Lippincott Williams and Wilkins, New York, USA.

5 Vipul N, Taif A, Joe J, and Lalit M (2009) Successful revascularization of near total amputation of the upper limb at the sultan qaboos hospital. Oman Med J 24: 44-48.

6 Yelena A (2014) Microsurgical Technique for $1 \mathrm{~mm}$ vessel end-to-end anastomosis. JOMI 3: 1-2.

7 Ashok RK, Rahul KP, Sushil N (2013) Unfavourable results in free tissue transfer. Indian J Plast Surg 46: 247-255.
8 Adrian R, Hywel D (2015) Key notes on plastic surgery, $2^{\text {nd }}$ edn, Wiley Blackwell Publishers, USA.

9 Josef EF (2001) Mastery of surgery. Lippincott Williams, Philadelphia, USA.

10 Henderson A (2006) Hand function in the child: Foundation of remediation. Mosby Elsevier, Missouri, USA.

11 Neto JQ, Carli AD, Nakamoto HA, Bersani G, Crepaldi BE, et al. (2015) Prognostic factors on survival rate of fingers replantation. Acta Orthopaedic Bras 23: 16-18.

12 Abdelgawad AA, Kanlic EM (2011) Orthopedic management of children with multiple injuries. J Trauma Acute Care Surg 70: 15681574.

13 Sabapathy SR, Bhardwaj P (2013) Secondary Procedures in Replantation. Semin Plast Surg 27: 198-204.

14 Kelleher JC (1982) Replantation and revascularization surgery in children. Plast Reconstr Surg 69: 579.

15 Salyapongse AN (2015) Extremity replantation: A clinical guide. Springer, New York, USA. 